\title{
Gravitational Wave Emission from a Bounded Source: the Nonlinear Regime
}

\author{
H. P. de Oliveira*t \\ Universidade do Estado do Rio de Janeiro \\ Instituto de Física - Departamento de Física Teórica \\ Cep 20550-013. Rio de Janeiro, RJ, Brazil \\ I. Damião Soares \\ Centro Brasileiro de Pesquisas Físicas \\ R. Dr. Xavier Sigaud, 150. CEP 22290-180 \\ Rio de Janeiro, RJ, Brazil \\ (Dated: November 12, 2018)
}

\begin{abstract}
We study the dynamics of a bounded gravitational collapsing configuration emitting gravitational waves, where the exterior spacetime is described by Robinson-Trautman geometries. The full nonlinear regime is examined by using the Galerkin method that allows us to reduce the equations governing the dynamics to a finite-dimensional dynamical system, after a proper truncation procedure. Amongst the obtained results of the nonlinear evolution, one of the most impressive is the fact that the distribution of the mass fraction extracted by gravitational wave emission satisfies the distribution law of nonextensive statistics and this result is independent of the initial configurations considered.
\end{abstract}

Robinson-Trautman (RT) metrics are the simplest known solutions of vacuum Einstein's equations which may be interpreted as representing an isolated gravitational radiating system [1]. The metric can be expressed as

$$
d s^{2}=\left(\lambda(u, \theta)+\frac{B(u)}{r}+2 r \frac{\dot{K}}{K}\right) d u^{2}+2 d u d r-r^{2} K^{2}(u, \theta)\left(d \theta^{2}+\sin ^{2} \theta d \varphi^{2}\right),
$$

where $r$ is an affine parameter defined along the shear-free null geodesics determined by the vector field $\partial / \partial r$, dot means derivative with respect to $u ; \lambda(u, \theta)=\frac{1}{K^{2}}-\frac{K_{\theta \theta}}{K^{3}}+\frac{K_{\theta}^{2}}{K^{4}}-\frac{K_{\theta}}{K^{3}} \cot \theta$ is the Gaussian curvature of the surfaces $(u=$ const, $r=$ const.). The geometry is nonstationary and axially symmetric, admitting the obvious Killing vector $\partial / \partial \varphi$. As a consequence of Einstein equations, we have the following evolution equation

$$
-6 m_{0} \frac{\dot{K}}{K}+\frac{\left(\lambda_{\theta} \sin \theta\right)_{\theta}}{2 K^{2} \sin \theta}=0 .
$$

In the above the subscript $\theta$ denotes derivatives with respect to $\theta$. Eq. (2) is denoted the RT equation, governs the dynamics of the gravitational field and will be the basis of our analysis of the gravitational wave emission processes in RT spacetimes. Formally speaking, it allows to evolve initial data $K(u, \theta)$ prescribed on a given null surface $u=u_{0}$ (except in the case $m_{0}=0$ ). A particular and important solution of the field equations is the Schwarzschild metric obtained when $K(u, \theta)=K_{0}=$ const, where $\lambda(u, \theta)=K_{0}^{-2}$ and the corresponding Schwarzschild mass is $M_{S c h w}=m_{0} K_{0}^{3}$. This expression will be of particular importance in our characterization of the mass function of the configuration. Concerning this point, we note that in general the function $M(u, \theta)=B(u) K^{3}(u, \theta)$ is invariant under the coordinate transformation that reduces $B(u)$ to a constant $-2 m_{0}$; also this transformation induces that the type-D $\mathcal{O}\left(1 / r^{3}\right)$ curvature scalar associated with the mass aspect of the spacetime has a $(u, \theta)$ dependence given exactly by $B K^{3}$.

Foster and Newman $[\underline{3}]$ exhibited the linearized solution of the field equations. We generalize their approach

\footnotetext{
* Regular Associated of The Abdus Salam International Centre for Theoretical Physics - ICTP, Trieste, Italy

${ }^{\dagger}$ Electronic address: oliveira@dft.if.uerj.br

‡Electronic address: ivano@cbpf.br
} 
in a rather distinct way in order to take into account the nonlinearities of the field equations. For this proposal, we shall consider the Galerkin projection method [4] in which we adopted the following decomposition

$$
K^{2}(u, x)=A_{0}^{2} \mathrm{e}^{Q(u, x)}=A_{0}^{2} \exp \left(\sum_{k=0}^{N} b_{k}(u) P_{k}(x)\right),
$$

where we have introduced a new variable $x=\cos \theta$ such that $-1 \leq x \leq 1 ; N$ is the order of the truncation, $b_{k}(u)$ are the modal coefficients, and we have adopted the Legendre polynomials $P_{k}(x)$ as the basis functions of the projective abstract space. The internal product defined in this abstract space is $\left\langle P_{j}(x), P_{k}(x)\right\rangle \equiv$ $\int_{-1}^{1} P_{j}(x) P_{k}(x) d x=\frac{2 \delta_{k j}}{2 k+1}$. The evolution equations for the modal coefficients $b_{k}(u)$ are derived in following way: we substitute (3) into the equation for $\lambda(u, x)$, both into (2), and finally projecting the resulting equation into each basis function $P_{k}(x)$. Then,

$$
\dot{b}_{n}(u)=\frac{(2 n+1)}{12 m_{0} A_{0}^{2}}\left\langle\mathrm{e}^{-Q(u, x)}\left[\left(1-x^{2}\right) \lambda^{\prime}\right]^{\prime}, P_{n}(x)\right\rangle .
$$

with $n=0,1,2 . ., N$. Therefore, the dynamics of RT spacetimes is reduced to this system of $(N+1)$ equations.

The initial conditions $b_{k}\left(u_{0}\right)$ are determined by the initial data $K\left(u_{0}, x\right) \equiv k(x)$ since $b_{j}\left(u_{0}\right)=\frac{2\left\langle\ln k(x), P_{j}\right\rangle}{\left\langle P_{j}, P_{j}\right\rangle}$, $j=0, . ., N$. We integrate numerically the set of equations (4) for distinct families $k(x)$ and the following basic features of the nonlinear dynamics of RT spacetimes emerged. (i) All modal coefficients tend to zero asymptotically; the exception is $b_{0}(u)$ that approaches to a constant value which we denote by $b_{0}(\infty)$. This asymptotic configuration actually corresponds to the Schwarzschild solution with mass $M_{\infty}=m_{0} A_{0}^{3} \exp \left(3 b_{0}(\infty) / 2\right)$. Indeed, such a feature feature is in agreement with previous analytical studies on RT spacetimes [5]. (ii) For small values of the initial mass of the distribution, $M_{\text {init }}$, more rapidly the Schwarzschild solution is settled down; in addition a large fraction of $M_{\text {init }}$ is lost in the process due gravitational wave extraction. On the opposite side, if $M_{\text {init }}$ is large, then the evolution of RT spacetime towards the asymptotic Schwarzschild final state is very slow. Correspondingly, the fraction of $M_{\text {init }}$ lost is very small. (iii) The invariant characterization of the presence of gravitational radiation can be done using the Peeling theorem. From this the complete pattern gravitational wave emission is obtained [2]. (iv) Finally, the last important feature is the relation between the fraction of mass extracted $\Delta=\frac{\left|M_{\text {init }}-M_{\infty}\right|}{M_{\text {init }}}$ versus the initial mass $M_{\text {init }}$. For any family $k(x)$ the mentioned relation satisfies the distribution provided by the nonextensive statistics $[\underline{6}]$.

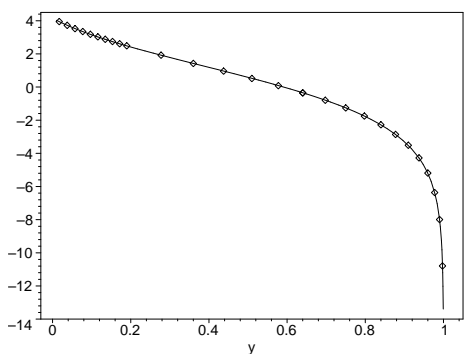

FIG. 1: Log-linear plot of $\Delta$ versus $M_{\text {init }}$. The solid line corresponds the nonextensive distribution $\Delta=C_{0}\left(y_{0}-\right.$ $y)^{\alpha}\left[1+(q-1) \lambda_{2}\left(y_{0}-y\right)\right]^{1 / 1-q}, y$ being associated to the initial mass, whereas the points were generated after integrating the dynamical system (4). The best fit is provided since $y_{0}=1, C_{0} \simeq 4.462, \alpha \simeq 1.992$ and $q \simeq 1.725$. The initial data are ellipses with distinct eccentricities.

The authors acknowledge the financial support of CNPq. H. P. de Oliveira is grateful to ICTP where this work was initiated.

[1] I. Robinson and A. Trautman, Phys. Rev. Lett. 4, 431 (1960); Proc. Roy. Soc. A265, 463 (1962). 
[2] H. P. de Oliveira and I. Damião Soares, Gravitational wave emission from a bounded source: a treatment in full nonlinear regime, (extended version) submitted to Physical Review D.

[3] J. Foster and E. T. Newman, J. Math. Phys. 8, 189 (1967).

[4] P. Holmes, John L. Lumley and Gal Berkooz, Turbulence, Coherent Structures, Dynamical Systems and Symmetry, Cambridge University Press (Cambridge, 1998).

[5] P. Chrusciel, Commun. Math. Phys. 137, 289 (1991); Proc. Roy. Soc. London 436, 299 (1992); P. Chrusciel and D. B. Singleton, Commun. Math. Phys. 147, 137 (1992).

[6] C. Tsallis, J. Stat. Phys. 52, 479 (1988). 